\title{
The US Real GNP is Trend-Stationary After All
}

\author{
Tolga Omay ${ }^{*}$, Rangan Gupta ${ }^{\star}$ and Giovanni Bonaccolto ${ }^{\#}$
}

\begin{abstract}
This paper applies the Fractional Frequency Flexible Fourier Form (FFFFF) Dickey-Fuller (DF)-type unit root test on the natural logarithm of US real GNP over the quarterly period of $1875: 1-2015: 2$, to determine whether the same is trend- or difference-stationary. While, standard and Integer Frequency Flexible Fourier Form (IFFFF) DF-type test fails to reject the null of unit root, the relatively more powerful FFFFF DF-type test provides strong evidence of the real GNP as being trend-stationary, i.e., US output returns to a deterministic log-nonlinear trend in the long run.
\end{abstract}

JEL Classification: C12; C22; E23.

Keywords: Fractional Frequency Flexible Fourier Form; Structural Break; Unit root; US real GNP

\footnotetext{
* Department of Management, Türk Hava Kurumu (THK) University, Bahçekapı Mahallesi Okul Sokak No:11, Ankara, Turkey. Email: tomay@thk.edu.tr.

- Corresponding author. Department of Economics, University of Pretoria, Pretoria, 0002, South Africa. Email: rangan.gupta@up.ac.za.

\# Department of Statistical Sciences, University of Padova, via C. Battisti 241, 35121 Padova, Italy. Email: bonaccolto@stat.unipd.it.
} 


\section{Introduction}

Nelson and Plosser (1982) challenged the accepted hypothesis of output being trendstationary, i.e., output returns to a deterministic loglinear trend in the long run. Instead, the authors provided statistical evidence that output is best viewed as difference-stationary, i.e., as a unit root process with drift. In other words, Nelson and Plosser (1982) suggested that shocks to real output have permanent rather than temporary effects. Following this piece of work by Nelson and Plosser (1982), a huge literature has emerged (for a detailed literature review, see for example, Murray and Nelson (2000), Camacho (2011), Shelley and Wallace (2011), Cushman (2012), Hosseinkouchack and Wolters (2013), Balcilar et al. (forthcoming) and references cited therein). This line of research applies different unit root tests to determine whether output of the US economy (measured either by real Gross Domestic Product (GDP) or real Gross National Product (GNP)) is trend-stationary or difference-stationary. More importantly, reading of the literature would indicate that it has not yet converged to a conclusive answer, with results contingent on tests and sample periods.

Against this backdrop, the objective of this study is to try and provide a definitive answer to this debate by analyzing the unit root property of an unique dataset of US real GNP, covering quarterly period of 1875:1-2015:2, which, to the best of our knowledge, is the longest possible data on US output available at quarterly frequency, i.e., the most relevant frequency at which output is measured globally. ${ }^{1}$ Given that we cover over 140 years of data, we use the recently developed powerful unit root test with multiple smooth structural breaks of Omay (2015), based on a Fractional Frequency Flexible Fourier Form (FFFFF) to determine whether real GNP of the US is trend- or difference-stationary.

Recently, multiple smooth breaks have been modeled by Flexible Fourier Transforms by Becker et al. (2006), Enders and Lee (2012 a, b), and Rodriques and Taylor (2012). The Fourier approach can capture the behavior of a deterministic function of unknown form even if the function itself is not periodic. Hence, it works better than dummy variable methods irrespective of whether the breaks are instantaneous or smooth, and avoiding the problems of selecting the dates, number and form of breaks (Omay, 2015). As Omay (2015) points out, the papers by Becker et al. (2006), Enders and Lee (2012 a, b), and Rodriques and Taylor (2012) indicated that, due to the problem of over-filtration, single frequency component of the Fourier Transforms should be used for structural break determination. However, following Becker et al. (2004), Omay (2015) considers the Fractional Frequency version of the Flexible Fourier Transform-based Dickey and Fuller (Augmented Dickey-Fuller (ADF), 1979)-type tests developed by Becker et al. (2006), Enders and Lee (2012 a, b), and Rodriques and Taylor (2012), to show that the small sample properties of the proposed test are found to be better than that of the integer frequency counterpart. Given this, we compare the results from the FFFFF of the standard ADF test with the Integer Frequency Flexible Fourier Form (IFFFF) of the DF test, and the basic ADF test without structural breaks, applied to the real GNP data of the US. To the best of our knowledge, this is the first such attempt based on the above-discussed three tests to try and analyze the unit root properties of the US quarterly output. The rest of the paper is organized as follows: Section 2 presents the basics of the ADF-type unit root test with FFFF; Section 3 discusses the data and the empirical findings, while Section 4 concludes.

\footnotetext{
${ }^{1}$ Annual data of U.S. real GDP is available from the year 1800 from the Global Financial Database.
} 


\section{Unit Root Test with Fractional Frequency Flexible Fourier Form}

The following equation is considered;

$$
y_{t}=d(t)+\phi_{1} y_{t-1}+\lambda t+\varepsilon_{t}
$$

where $\varepsilon_{t}$ is a stationary disturbance with variance $\sigma^{2}$, and $d(t)$ is a deterministic function of $t$. We also note that the initial value of $y_{t}$, i.e. $y_{0}$, is assumed to be a fixed value, and $\varepsilon_{t}$ is weakly dependent as in Enders and Lee (2012 a, b). As pointed out by Enders and Lee (2012 a, b), and Omay (2015), if the functional form of $d(t)$ is known, it is possible to estimate Eq. (1) and to test the null hypothesis of a unit root. Further, when the functional form of $d(t)$ is unknown, any test for $\phi_{1}=1$ is difficult if $d(t)$ is miss-identified. The tests of Enders and Lee (2012 a, b) and Omay (2015) approximates $d(t)$ by employing the Fourier expansion as follows:

$$
d(t)=\alpha_{0}+\alpha_{k} \sin \left(\frac{2 \pi k t}{T}\right)+\beta_{k} \cos \left(\frac{2 \pi k t}{T}\right)
$$

where $k$ indicates a particular frequency, and $T$ is the number of observations. When there is no nonlinear trend, all values of $\alpha_{k}=\beta_{k}=0$, which in turn, yields the ADF test as a special case.

As recommended in the literature, a specific single frequency often leads to a good approximation to a model with structural change, and hence, we do the same. Recall that, we use the fractional frequency instead of integer ones as stated in the introduction. For selecting the best fitting fractional single frequency, we follow the completely data-driven procedure of Davies (1987). The grid search method works as follows: we run a regression by using Equation (2), with the single frequency between the intervals $0.1 \leq k^{f r} \leq k_{\max }^{f r}$; we set $k^{\max }=2$ as recommended in Enders and Lee (2012 a, b) and Omay (2015). However, for fractional frequencies, we select $k=0.1$ as increments of the selected frequencies. Finally, we obtain the specific (optimal) $k=\hat{k}^{f r}$ that minimizes the sum of square residuals of Equation (2). Formally, the testable regression is:

$$
\Delta y_{t}=\rho y_{t-1}+c_{1}+c_{2} t+c_{3} \sin \left(\frac{2 \pi k^{f r} t}{T}\right)+c_{4} \cos \left(\frac{2 \pi k^{f r} t}{T}\right)+e_{t}
$$

\section{Data and empirical application}

In this study, we use data on the real US GNP covering the period quarterly of $1875: 1$ to 2015:2 ( $T=562$ ), with the start and end dates being purely driven by data availability. Our data on the nominal GNP and the GNP deflator, with the latter used to deflate the former to yield real values, are derived from two sources. First, the observations covering the period 1875:11946:4 are obtained from National Bureau of Economic Research (NBER), available for download at: http://www.nber.org/data/abc/; the actual sources are the tables of quarterly data corresponding to Appendix B of Gordon (1986). To the best of our knowledge, this is the only existing source for the pre-1947 quarterly data on US GNP and GNP deflator, with National Income and Product Account (NIPA) quarterly data series non-existent before 1947. Second, data from 1947:1-2015:2 is sourced from the FRED database of the Federal Reserve Bank of 
St. Louis. Note that, the dataset compiled by Gordon (1986) runs till 1983:4, with the base year of the GNP deflator being 1972. Given that nominal GNP and GNP deflator data based on the NIPA are available from 1947:1, we decided to use, for those variables, the FRED database, rather than the Gordon (1986) one, which, in any case, would have ran only till 1983:4. The base year of the GNP deflator for the period 1875:1-1946:4 is updated from 1972 to 2009 to correspond to the base year of the GNP deflator based on the NIPA, so that the real GNP is ultimately in constant 2009 prices. For our various unit root tests, we work with the natural logarithm of the real GNP data (LGNP). The data has been plotted in Figure A1 in the Appendix of the paper.

Besides the FFFFF DF-type test of Omay (2015), Table 1 also reports the IFFFF DF-type test of Enders and Lee (2012a, b), and the standard DF test. Not surprisingly, the standard DF test fails to reject the null of unit root with a constant and trend in the test-equation. The IFFF DFtest (with $k=2$ ) too fails to reject the null of unit root in LGNP at the conventional (5\%) level of significance. The test does, however, provide weak evidence of trend-stationarity of the LGNP at the $10 \%$ level of significance. ${ }^{2,3}$ Next, we turn to the FFFFF DF-type test of Omay (2015).

For detailed investigation of the optimal frequency $\left(\hat{k}^{f r}\right)$, we have used $0.1,0.01$, and 0.001 increments of the fractional frequencies to obtain 1.7, 1.670, and 1.667 values of the same, respectively. In all these cases, the null of unit root of the LGNP is rejected at the 5\% level of significance, with the most significant test value being at $k^{f r}=1.667$. Figure A1 plots the LGNP along with the estimated LGNP with $k^{f r}=2.0$ and $k^{f r}=1.667$, while Figure A2 plots the residual from the corresponding fits of LGNP, i.e., the residual after detrending LGNP with a linear trend and Fourier intercepts under $k^{f r}=2.0$ and $k^{f r}=1.667$. As can be seen from Figure A1, the fits are quite similar under the integer and the fractional cases, with both the estimates missing the sharp breaks during the "Great Depression”. Figure A2, provides an alternative picture of the fits in terms of the residuals, which tend to differ towards the latter half of the sample. As indicated by Omay (2015), it is reasonable to use only the 0.1 increments, because the other frequencies which are obtained for smaller increments are all clustered around this fractional frequency $k^{f r}=1.7$, with the power loss not exceeding $1 \%$. Hence, using this 0.1 increment for fractional frequency has the possibility of limiting type-two errors, overfiltration, and inappropriate nonlinear trend problems. In any event, more importantly, while the IFFFF and standard DF-type tests fail to reject the null hypothesis of unit root test, the more powerful FFFFF DF-type test indicates that real GNP of the US is, in fact, (nonlinear) trend-stationary, and not difference-stationary as posited by Nelson and Plosser (1982). ${ }^{4}$

\footnotetext{
2 In an earlier version of the Enders and Lee (2012a) paper, available at: http://www3.nd.edu/ meg/MEG2004/Lee-Junsoo.pdf, the authors also failed to reject the null of unit root in real GDP of the US over the period of 1947:1-2003:2.

${ }^{3}$ Using standard unit root test with one break (Zivot and Andrews, 1992) and two breaks (Lumsdaine and Papell, 1997; Lee and Strazicich, 2003) in both the mean and the trend, we could not reject the null of unit root even at $10 \%$ level of significance. Complete details of these results are available upon request from the authors.

${ }^{4}$ In a related area of research, many studies (Hamilton, 1989; Perron, 1989; Balke and Fomby, 1991; Beaudry and Koop, 1993; Murray and Nelson, 2000; Kim et al., 2005; Camacho, 2011; and Hosseinkouchack and Wolters, 2013) have accounted for the possibility that the persistence of the US output might differ in recessions and expansions. Not surprisingly, the evidence is, at best, mixed, with results depending on the tests and sample period used. Given this, following Hosseinkouchack and Wolters (2013), we tested the unit root hypothesis not only at the conditional mean of the real GNP, but also in the tails of the distribution using a quantile autoregression (QAR)-based unit root test. Our results, based on the quantile Kolmogorov-Smirnov (QKS) test of Koenker and Xiao (2004), which checks for the unit root property across a range of quantiles, corroborated the findings of the FFFFF DF-type test. In other words, the QKS test confirmed that LGNP is trend-stationary, with the results being driven by the quantiles ranging from 0.45 to 0.95 at $5 \%$ level of significance. Specifically,
} 
Table 1. Unit root tests on US real GNP (1875:1-2015:2)

\begin{tabular}{|c|c|c|c|c|c|}
\hline \multirow[b]{2}{*}{$k$} & \multirow{2}{*}{$\begin{array}{c}\tau_{D F_{-} \tau} \\
2.0\end{array}$} & \multicolumn{4}{|c|}{$\tau_{D F_{-} \tau}^{f r}$} \\
\hline & & 1.70 & 1.670 & 1.667 & $D F_{\tau}$ \\
\hline & $-3.828 *$ & $-4.322 * *$ & $-4.338 * *$ & $-4.339 * *$ & -3.075 \\
\hline
\end{tabular}

Notes: The lag-length is 10 as selected by the Akaike Information Criterion (AIC) with a maximum lag set at 18; $D F_{\tau}, \tau_{D F_{-} \tau}$ and $\tau_{D F_{-} \tau}^{f r}$ corresponds to the ADF test with a constant and trend, the Enders and Lee (2012a) test and the Omay (2015) test respectively. $10 \%, 5 \%$, and $1 \%$ critical values of the $D F_{\tau}$ test are: $-3.131,-3.418$ and 3.975; $10 \%, 5 \%$ and $1 \%$ critical values of the $\tau_{D F_{-} \tau}$ test for $k=2$ are: $-4.578,-3.985$ and $-3.676 ; 10 \%, 5 \%$ and $1 \%$ critical values of the $\tau_{D F_{-} \tau}^{f r}$ test at $k^{f r}=1.70$ are: $-3.830,-4.140$ and $-4.700 ; * *(*)$ indicates rejection of the null of unit root at 5\% (10\%) level of significance.

\section{Concluding remarks}

This paper aims to provide a definitive answer to the unresolved question of whether US output is best characterized by a trend- or difference-stationary process. To achieve our purpose, we apply the recently developed powerful unit root test with multiple smooth structural breaks of Omay (2015), based on a Fractional Frequency Flexible Fourier Form (FFFFF) on a unique data of US real GNP covering the quarterly period of 1875:1-2015:2. We find that, while, the standard Dickey and Fuller (DF, 1979) and the Integer Frequency Flexible Fourier Form (IFFFF) of the DF test developed by Enders and Lee (2012a, b) fails to reject the null of unit root, the FFFFF DF-type test provides strong evidence of the natural logarithm of real GNP as being trend-stationary. In other words, our paper provides evidence that the US real GNP returns to a deterministic log-nonlinear trend (as characterized by smooth structural shifts) in the long run.

\footnotetext{
while recessions are found to be permanent, the results suggested that expansions are temporary. Next, when we filtered the LGNP data with the linear trend and the Fourier intercept, and then applied the quantile unit root test, the QKS test again confirmed that LGNP is trend-stationary. While, stationarity is found to hold for quantiles 0.45 to 0.95 (at $5 \%$ level of significance) under $k^{f r}=2.0$, the same holds for a bigger part of the distribution ( 0.35 to 0.95 at $5 \%$ level of significance) with $k^{f r}=1.667$. In addition, we also used the $D Q$ and $S Q_{\tau}$ tests of Qu (2008) and Oka and Qu (2011) to check for breaks in the entire conditional distribution and at specific quantiles of the conditional distribution, respectively. For the entire conditional distribution, we obtained two breaks at 1903:2 and 1952:3, with the breaks primarily originating from the median of the distribution - the latter result being an indication of two regimes in the LGNP process. When we filtered the LGNP data with a linear trend and the two breaks (captured by dummy variables), we found that LGNP is trend-stationary over the quantile range of 0.35 to 0.95 at $5 \%$ level of significance. Complete details of these results are available upon request from the authors. Note that, our results are different from those obtained by Hosseinkouchack and Wolters (2013), whereby these authors indicated that null of unit root cannot be rejected over the entire conditional distribution of the US output (measured by real GDP over 1947:1-2012:1). However, these authors did not account for breaks in the conditional distribution of the real GDP.
} 


\section{References}

Balcilar, M., Gupta, R., Josste, C., and Ranjbar, O. (Forthcoming). Characterising the South African Business Cycle: Is GDP Difference-Stationary or Trend-Stationary in a MarkovSwitching Setup? Economia Internazionale.

Balke, N. and Fomby, T. (1991). Shifting trends, segmented trends, and infrequent permanent shocks. Journal of Monetary Economics 28 (1), 61-85.

Beaudry, P. and Koop, G. (1993). Do recessions permanently change output? Journal of Monetary Economics 31, 149-163.

Becker R, Enders W., Hurn, S. (2004). A General Test for Time Dependence in Parameters. Journal of Applied Econometrics 19, 899-906.

Becker R, Enders W., Lee, J. (2006). A Stationarity Test in The Presence of an Unknown Number of Smooth Breaks. Journal of Time Series Analysis 27, 381-409.

Camacho, M. (2011). Markov-switching models and the unit root hypothesis in real U.S. GDP. Economics Letters 112: 161-164.

Cushman, D. O. (2012). Mankiw vs. DeLong and Krugman on the CEA's real GDP forecasts in early 2009: What might a time series econometrician have said? Econ Journal Watch 9(3), 309-349.

Davies, R.B. (1987). Hypothesis testing when a nuisance parameter is only identified under the Alternative, Biometrika, 47, 33-43.

Dickey, D. A. and Fuller, W. A. (1979). Distribution of the estimators for autoregressive time series with a unit root, Journal of the American Statistical Association, 74, pp. 427-31.

Enders, W., Lee, J. (2012a). A Unit Root Test Using a Fourier Series to Approximate Smooth Breaks. Oxford Bulletin of Economics and Statistics 74(4), 574-599.

Enders, W., Lee, J. (2012b). The Flexible Fourier Form and Dickey-Fuller type Unit Root Tests. Economic Letters, 117, 196-199.

Gordon, R.J. (1986). The American Business Cycle: Continuity and Change. University of Chicago Press.

Hamilton, J.D. (1989). A new approach to the economic analysis of nonstationary time series and the business cycle. Econometrica 57(2), 357-384.

Hosseinkouchack, M. and Wolters, M. (2013). Do large recessions reduce output permanently? Economics Letters, December 2013, 121(3), 516-519.

Koenker, R. and Xiao, Z. (2004) Unit root quantile autoregression inference, Journal of the American Statistical Association, 99, 775-87. 
Kim, C.-J., J. Morley, and Piger, J. (2005). Nonlinearity and the permanent effects of recessions. Journal of Applied Econometrics 20(2), 291-309.

Lee, J., and Strazicich, M. C., (2003). Minimum LM Unit Root Test with Two Structural Breaks. Review of Economics and Statistics 85, 1082-1089.

Lumsdaine, R., and Papell, D., (1997). Multiple Trend Breaks and the Unit Root Hypothesis. Review of Economics and Statistics 79, 212-218.

Murray, C. and Nelson, C. (2000). The uncertain trend in US GDP. Journal of Monetary Economics 46 (1), 79-95.

Oka, T. and Qu, Z. (2011). Estimating structural changes in regression quantiles. Journal of Econometrics 162, 248-276.

Omay, T. (2015). Fractional Frequency Flexible Fourier Form to approximate smooth breaks in unit root testing. Economics Letters 134, 123-126.

Qu, Z. (2008). Testing for structural change in regression quantiles. Journal of Econometrics $146,170-184$.

Rodriques, P., Taylor, A.M.R. 2012. The Flexible Fourier Form and Local generalised least Squares De-trended Unit Root Tests. Oxford Bulletin of Economics and Statistics, 74(5), 736759.

Shelley, G. L. and Wallace, F.H. (2011). Further evidence regarding nonlinear trend reversion of real GDP and the CPI. Economics Letters 112(1), 56-59.

Zivot, E., Andrews, D. W. K., (1992). Further Evidence on the Great Crash, the Oil Price Shock and the Unit Root Hypothesis. Journal of Business and Economic Statistics 10, 251270. 


\section{APPENDIX:}

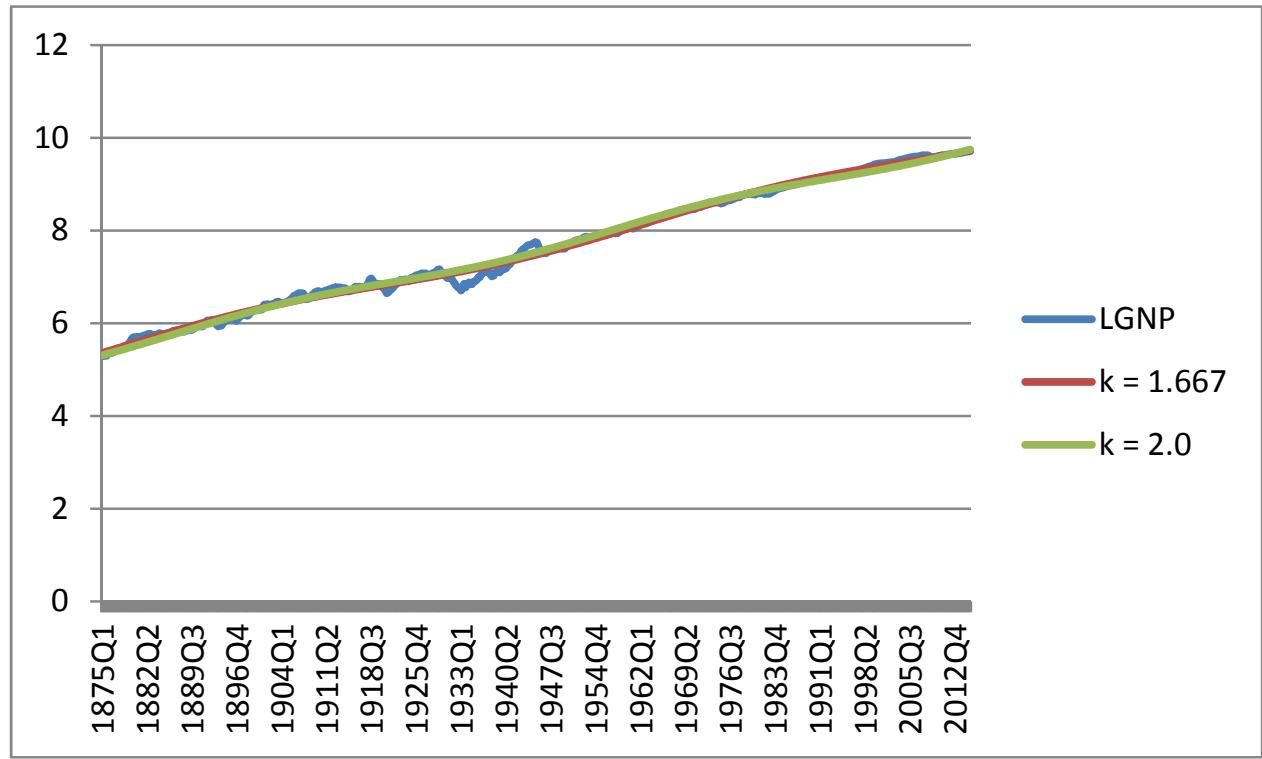

Figure A1. Plot of the natural logarithms of US real GNP (LGNP) and fits under integer $(k=2.0)$ and fractional $(k=1.667)$ Fourier functions

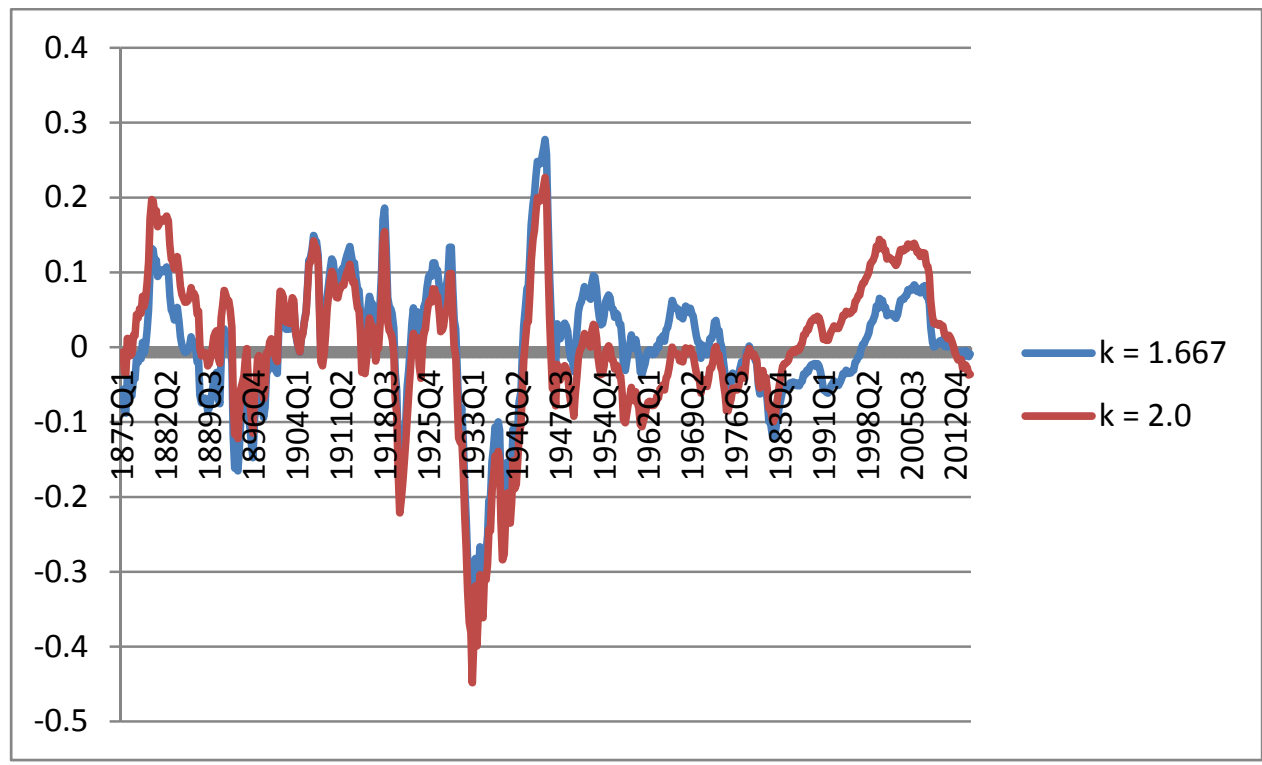

Figure A2. Plot of residuals after detrending with a linear trend and integer $(k=2.0)$ and fractional $(k=1.667)$ Fourier intercepts 